\title{
Ärztenetzwerke im Kanton Bern - nur Hoffnung oder die Realität von morgen?
}

K. W. Matter-Walstra ${ }^{a}$, B. Künzi ${ }^{b}$

a Dr. phil. nat., Institut für evaluative Forschung IEFO, Bern

b Dr. med., Wissenschaftlicher Leiter swisspep Institut für Qualität und Forschung im Gesundheitswesen, Gümligen
Korrespondenz:

Dr. med. Beat Künzi

Facharzt für Allgemeinmedizin FMH und für Psychosomatik APPM swisspep Institut für Qualität und Forschung im Gesundheitswesen CH-3073 Gümligen

beat.kuenzi@swisspep.ch
Viele Ärzte, aber ebenso Versicherte, Patienten und Politiker, etwas weniger auch die Versicherer setzen grosse Hoffnungen in Managed Care und speziell in Ärztenetzwerke zur Erhaltung einer qualitativ guten und finanzierbaren medizinischen Versorgung. Inwieweit die Ärzteschaft diese Einschätzungen und Hoffnungen teilt, wurde nun erstmals systematisch untersucht. Dieser Artikel fasst die wichtigsten Resultate einer unabhängigen und repräsentativen Gesamtbefragung unter dem Titel «Meinungen und Verhalten der Berner Ärzteschaft gegenüber Ärztenetzwerken» zusammen. Die Studie wurde im Auftrag der Ärztegesellschaft des Kantons Bern und mit der Finanzierung durch das Trust Center PonteNova (das auch die Betriebsorganisation für Ärztenetzwerke ist) vom swisspep In-

\section{Réseaux médicaux dans le canton de Berne: seulement un espoir} ou la réalité de demain?

De nombreux médecins, mais aussi des assurés, des patients et des politiques (un peu moins les assureurs), placent de grands espoirs dans le managed care (gestion des soins) et principalement dans les réseaux de médecins pour conserver une assistance médicale de bonne qualité et financièrement supportable. Pour savoir dans quelle mesure le corps médical partage cette vision des choses et ces espoirs, une enquête systématique a été menée pour la première fois. Le présent article rassemble les résultats les plus importants de cette enquête globale indépendante et représentative intitulée «Opinions et attitude de la Société des médecins du Canton de Berne à l'égard des réseaux médicaux». L'étude a été menée en automne 2006 sur mandat de la Société des médecins du Canton de Berne par I'Institut swisspep pour la qualité et la recherche en santé publique en collaboration avec l'Université de Berne (Institut pour la recherche évaluative IEFO), avec le financement du centre fiduciaire PonteNova stitut für Qualität und Forschung im Gesundheitswesen in Zusammenarbeit mit der Universität Bern (Institut für evaluative Forschung IEFO) im Herbst 2006 durchgeführt.

Ein Drittel der Ärzte mit Praxisbewilligung im Kanton Bern ist Mitglied in einem strukturierten Ärztenetzwerk, und mindestens weitere 50\% gedenken in den nächsten Jahren beizutreten. $16,5 \%$ lehnen diese explizit ab. Netzwerkmitglieder beurteilen alle erfragten Aspekte von Ärztenetzwerken am positivsten, gefolgt von jenen Kolleginnen und Kollegen, die in den nächsten zwei Jahren beitreten werden. Die grössten Zweifel bezüglich negativer Auswirkungen von Ärztenetzwerken betreffen die Qualität der Patientenbetreuung, während Massnahmen zur Qualitätssicherung und finanzielle Auswir-

(qui se trouve être aussi l'organisation de gérance des réseaux médicaux).

Un tiers des médecins avec autorisation de pratiquer dans le canton de Berne sont membres d'un réseau médical structuré et un autre $50 \%$ d'entre eux au moins songent à faire le pas dans les prochaines années. $16,5 \%$ des médecins bernois consultés rejettent l'idée. Ce sont les membres de réseaux qui émettent les avis les plus positifs sur l'ensemble des aspects abordés par le questionnaire, suivis par leurs consœurs et confrères qui ont décidé d'adhérer ces deux prochaines années. Les plus grands doutes exprimés à l'encontre des réseaux médicaux concernent la qualité de la prise en charge des patients, alors que les mesures d'assurance-qualité et les effets financiers ont tendance à être appréciés de façon neutre. Cela dit, la question de la coresponsabilité budgétaire constituera une véritable pierre de touche pour les réseaux médicaux. 
kungen tendenziell neutral beurteilt werden. Die Übernahme von Budgetmitverantwortung wird allerdings zum Prüfstein von Ärztenetzwerken werden.

\section{Hintergrund}

Optimal vernetzt arbeitende Einzelpersonen und Organisationen führen zu wirtschaftlichen Strukturen mit deutlich besserer Wertschöpfung [1]. Steigende Gesundheitsausgaben durch zunehmende Nachfrage und immer komplexere Leistungsangebote lassen strategische Partnerschaften unter Leistungserbringern wichtiger werden. Netzwerkmitarbeiter sind - im Gegensatz zu nichtvernetzten - produktiver und zufriedener, auch weniger in Konflikte verstrickt [2]. Obwohl sich Ärztenetzwerke (ÄNW) aus dieser Perspektive als eine der Möglichkeiten zur Lösung der Probleme in Gesundheitswesen anbieten, wurde deren Struktur, Funktionsweise und Resultate bisher wenig empirisch erforscht [1]. Erschwerend ist dabei, dass sich die Definition, Organisation und Zielsetzungen von ÄNW von Land zu Land und je nach Ausgangssituation unterscheiden [3-6]. Verschiedene Organisationsformen von ÄNW können und müssen von üblichen Formen ärztlicher Zusammenarbeit, aber auch von reinen Disease-ManagementProgrammen unterschieden werden: einerseits das sogenannte «Shared Care»-Modell, das erstmals von der Gruppe um Wagner-von Korff $[7,8]$ als «Chronic Care Model» oder «Collaborative Interventions»-Modell beschrieben wurde, und andererseits «Integrierte Versorgungsmodelle», in denen Grundversorger mit Spitälern als multidisziplinäre Teams kooperieren [9] und/ oder Budget(mit)verantwortung übernehmen, wie beispielsweise in sogenannten ManagedCare-(MC-)Modellen [10] oder in «fund holding»-Praxen $[11,12]$. Aus organisatorischer Sicht können ÄNW mit einer «top-down»-Struktur, wie etwa die angloamerikanischen «Health Plans», von demokratischeren und durch Ärzte initiierte «bottom-up»-Lösungen unterschieden werden, wie sich diese nun in der Form der ÄNW in der Schweiz beobachten lässt.

Obwohl für verschiedene Organisationsformen von ÄNW bisher positive Auswirkungen auf patientenrelevante Resultate nachgewiesen wurden [13-15], sollten auch mögliche Nachteile im Auge behalten werden, wie etwa eine höhere Arbeitsbelastung [16] oder fragwürdige Strategien zur Kostenreduktion [17], neben fehlenden Auswirkungen auf die Überweisungsraten oder die Betreuungsqualität $[11,12]$. In Ländern wie der Schweiz, in denen der Arzt als unabhängiger Kleinunternehmer wirkt, kann auch der Rollen- wechsel - zum eingebundenen ÄNW-Mitglied mit definierten Rechten und Pflichten - als Autonomieverlust und somit als bedrohlich erscheinen [18]. Bell hat bereits vor zehn Jahren die Einstellungen der US-amerikanischen Ärzte zu Managed Care untersucht und festgestellt, dass eine Mehrheit der Ärzte die Betreuungsqualität in MC-Modellen als Problem sieht, gleichzeitig aber MC als unabwendbare Entwicklung einschätzt, auf die sich Ärzte - längerfristig einlassen sollten oder müssen [19].

In der Schweiz wurden MC-Modelle in verschiedenen Formen umgesetzt: Ein erster prospektiver Versuch einer «Health Maintenance Organization» (HMO) wurde 1990 gestartet [20]. Später wurden Hausarztnetzwerke unter folgender Definition gegründet: Organisationen von Leistungserbringern, die sich einer gemeinsamen Betreuungskultur verpflichten, basierend auf vereinbarten Behandlungsprozessen und unternehmerischen Organisationsstrukturen. ÄNW setzen sich bei hoher Versorgungsqualität einen haushälterischen Umgang mit den vorhandenen Mitteln zum Ziel; Praktikerqualitätszirkeln kommt dazu eine zentrale Bedeutung zu. ÄNW können mit oder ohne Budgetmitverantwortung arbeiten und auch mehr oder weniger konsequent einer Risikoselektion vorbeugen, primär durch eine hohe Abdeckung [21]. Die Mitgliedschaft in einem ÄNW ist in der Schweiz, im Gegensatz etwa zur Situation in Grossbritannien [3], freiwillig. Allerdings zeigen die Diskussionen rund um die Aufhebung des Kontrahierungszwanges, dass die Mitgliedschaft in einem ÄNW für den einzelnen Arzt in Zukunft einen anderen Stellenwert erhalten könnte: Die FMH lehnt die Aufhebung individueller Kontrahierung bekanntlich mit dem Argument ab, dass besser MC-Modelle und speziell ÄNW weiterentwickelt werden sollten und sich allenfalls in Verträgen mit ÄNW «Elemente einer differenzierten Kontrahierung» realisieren liessen [22].

Bezüglich der Ausgestaltung von ÄNW hat sich der Vorstand der Berner Ärztegesellschaft hinter das «Positiv-Modell» des VSAO gestellt, das eine Budgetverantwortung klar ablehnt [23]. Angesichts der vielen offenen Fragen im Zusammenhang mit ÄNW stellt sich nun erstmals der gesamten Ärzteschaft die Frage, wie solche zukünftigen ÄNW zur Erzielung einer qualitativ hochstehenden und kosteneffizienten Betreuung auszugestalten wären und in welcher Art die niedergelassenen Ärzte an diesen Entwicklungen teilnehmen (werden). Es ist das Verdienst der Berner Ärztegesellschaft - zusammen mit dem Trust Center PonteNova und PonteNet -, die eine erste repräsentative Studie unter dem Titel «Mei- 
nungen und Verhalten der Berner Ärzteschaft gegenüber ÄNW» in der Schweiz in Auftrag gab. Diese Organisationen wollen die Bildung von ÄNW unterstützen, die Studie sollte zeigen, wie und wo.

\section{Material und Methoden}

\section{Fragebogenentwicklung}

Für diese Studie wurde ein Fragebogen, basierend auf einer systematischen Literatursuche und unterstützt durch ein Panel aus nationalen Experten und Ärzten mit und ohne MC-Erfahrung, entwickelt (RAND-Panel-Methode [24]). Daraus resultierten 36 geschlossene und 3 offene Fragen zu Einstellungen, Erfahrungen oder Meinungen und erwarteten Auswirkungen von ÄNW mit und ohne Budgetmitverantwortung auf die Patientenbetreuung, Finanzen und Arbeitsbelastung. Ergänzend wurden Fragen zur Qualitätssicherung innerhalb von ÄNW und zur Übernahme von Verantwortung im Gesundheitswesen gestellt. Schliesslich sollten Fragen zur bisherigen Erfahrung mit ÄNW oder zur Beitrittsbereitschaft zusammen mit den demographischen Angaben eine stratifizierte Auswertung erlauben. Der Fragebogen wurde in einer Gruppe von 20 zufällig ausgewählten Mitgliedern der Berner Ärztegesellschaft pilotiert.

Die geschlossenen Fragen zu Einstellungen, Erfahrungen oder Meinungen und erwarteten Auswirkungen konnten mit fünfstufigen Antworten beantwortet werden (je nach Frage verschieden formuliert: negativ/positiv, Zustimmung/Ablehnung, schlechter/besser), mit dem Wert 1 für sehr negative, 2 für eher negative, 3 für neutrale, 4 für eher positive und 5 für sehr positive Einstellung/Erfahrungen/Auswirkungen.

\section{Teilnehmer}

Die Umfrage wurde allen Ärzten mit einer Praxisbewilligung im Kanton Bern $(n=1961)$ einschliesslich der Spitalärzte und Belegärzte zugestellt. Ausgeschlossen wurden Pathologen $(n=3)$, Radiologen ( $\mathrm{n}=20)$ und Anästhesisten $(\mathrm{n}=55)$. Der Versand der Umfrage erfolgte Ende September 2006, mit zwei Erinnerungsaktionen (Ende Oktober per E-Mail, im November mit Brief).

\section{Datenauswertung und Statistik}

Zur Vereinfachung der Auswertung des umfangreichen Fragebogens wurden 30 der 36 Fragen mit Hilfe einer Faktorenanalyse in Dimensionen zusammengefasst. Die dazu berücksichtigten 30 Fragen betrafen Einstellungen gegenüber ÄNW und Meinungen zum Gesundheitswesen; die Fragen zur beruflichen Zufriedenheit wurden ausgeschlossen. Die gefundenen Dimensionen wurden anhand von Cronbach-Alpha-Analysen verifiziert. Zur Erleichterung einer eindeutigen Interpretation wurde nur der Anteil der beiden positivsten der fünf Antwortkategorien eingerechnet (Werte 4 oder 5, je nach Frage unterschiedlich formuliert), also «eher positiv» oder «sehr positiv». Dann wurde für jede Dimension der Anteil Antworten mit positiver oder zustimmender Meinung berechnet. Schliesslich wurden die Resultate entsprechend dem klinischen Hintergrund (Hausarzt, Spezialist sowie «andere», d.h. Spezialisten mit direktem Patientenzugang, wie Psychiater, HNO, Dermatologen usw.) und der Mitgliedschaft in einem strukturierten ÄNW (aktiv; vorgesehen: innert 2 Jahren, nach 2 Jahren, nie) untersucht. Die Zustimmung pro Dimension wurde nach ÄNW-Mitgliedschaft oder Funktion (Hausarzt, Spezialist usw.) stratifiziert mittels Global-Linear-Modellen (GLM) errechnet, die eine Korrektur für Alter und Geschlecht erlauben. Die statistischen Tests wurden mit SAS 9.1 ${ }^{\circledR}$ (SAS Institute Inc., Cary, NC, USA) durchgeführt; die Signifikanzschwelle bei wurde dabei auf $\mathrm{p}<0,05$ festgelegt.

\section{Resultate}

\section{Teilnehmer}

Von den 1961 versandten Fragebogen waren 156 nicht zustellbar oder falsch zugeordnet. 881 von den somit verbleibenden 1805 Fragebogen wurden retourniert, was einem Rücklauf von $48,8 \%$ entspricht. Je 75 Fragebogen waren leer wegen Praxisaufgabe bzw. Ablehnung der Teilnahme, auf 11 weiteren fehlte die Information bezüglich ÄNW-Mitgliedschaft. Somit standen schliesslich die Meinungen von 720 Kolleginnen und Kollegen zur Auswertung zur Verfügung (39,9\% des effektiv befragten Kollektivs).

Unter den Antwortenden waren 83,2\% ( $\mathrm{n}=599)$ Ärzte und 16,8\% (n=121) Ärztinnen, was den Geschlechteranteilen im befragten Kollektiv entspricht. Grundversorger waren anteilsmässig mit 59,2\% $(\mathrm{n}=426)$ übervertreten $(50 \%$ im befragten Kollektiv), die Konsiliarärzte mit $13,2 \%(n=95)$ leicht untervertreten $(16 \% \mathrm{im}$ befragten Kollektiv) und die $25 \%(n=183)$ «anderen» Spezialisten (mit in der Regel direktem Patientenzugang: Gynäkologen, Psychiater, Dermatologen, Ophthalmologen: 26\% im befragten Kollektiv) fast korrekt vertreten. 16 Teilnehmer gaben ihre Funktion nicht an. Der Anteil der retournierten und auswertbaren Fragebogen erreichte unter den 905 befragten Grundversorgern mit $47,4 \%$ den höchsten Wert, der entsprechende Anteil war unter den 470 «anderen» 
Ärzten $40 \%$ bzw. unter den 291 Konsiliarärzten $33,7 \%$. Die Fachärzte für Allgemeinmedizin FMH stellten mit 35\% die grösste Fachgruppe unter den Teilnehmern, die Gruppe der «anderen» Spezialisten umfasste zusammen 36\%, Internisten $23 \%$, Pädiater $4 \%$ und Nichttitelträger $2 \%$.

$30,0 \%(\mathrm{n}=216)$ der Teilnehmer waren bereits Mitglied in einem ÄNW (mit oder ohne Budgetmitverantwortung), nur $7 \%(n=51)$ sind bereits Mitglied oder im Aufnahmeprozess eines ÄNW mit Budgetmitverantwortung. 30,5\% $(\mathrm{n}=219)$ beabsichtigen, in den nächsten 2 Jahren und

\section{Abbildung 1}

Netzwerkmitgliedschaft nach Alter, Geschlecht und FMH-Titel oder Funktion.

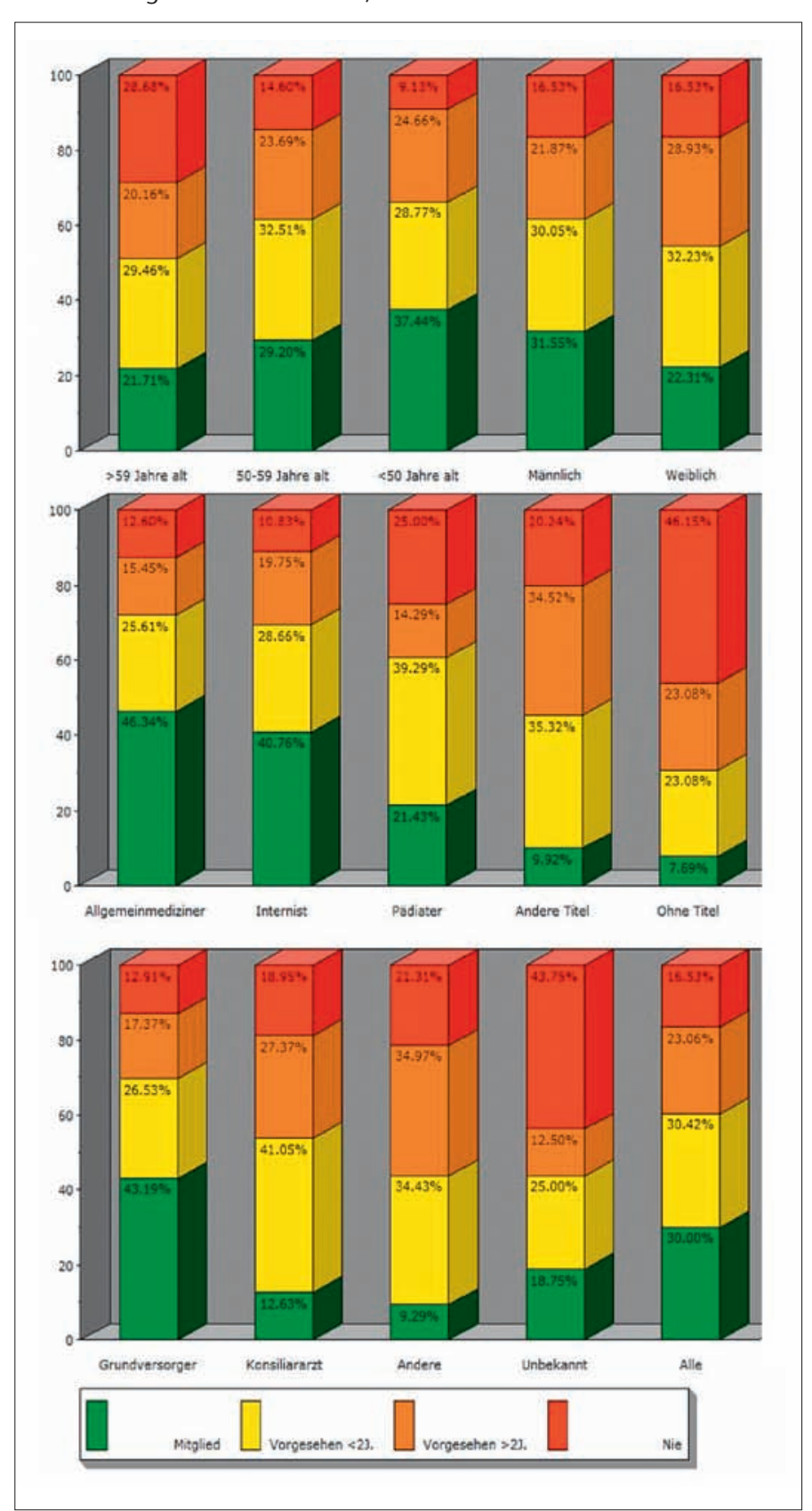

weitere $23 \%(\mathrm{n}=166)$ nach mehr als zwei Jahren einem ÄNW mit oder ohne Budgetverantwortung beizutreten. $16,5 \%(n=119)$ schlossen einen Beitritt zu einem ÄNW für sich aus («ÄNWGegner» Abb. 1). Der Beitrittswunsch bezieht sich allerdings mehrheitlich auf ÄNW ohne Budgetmitverantwortung, da 62,1\% der Beitrittswilligen ein ÄNW ohne solche Verpflichtung bevorzugen. «ÄNW-Gegner», d.h. Kollegen, die für sich einen Beitritt definitiv ausschliessen, sind mit einem Altersdurchschnitt von 55,8 Jahren signifikant älter, sowohl als ÄNW-Mitglieder mit einem Durchschnittsalter von 51,8 Jahren wie auch die Beitrittswilligen (mit 53,2 Jahren für jene, die in den nächsten zwei Jahren und 52,6 Jahre für jene, die nach zwei Jahren beitreten möchten). 96,7\% der «ÄNW-Gegner» lehnen auch Budgetmitverantwortung ab. Die höchsten Anteile an «ÄNW-Gegnern» fanden sich - nach Facharzttitel - unter Nichttitelträgern (46\%), Pädiatern (25\%) und Spezialisten (20\%). Demgegenüber fanden sich unter Grundversorgern nur $13 \%$ «ÄNW-Gegner».

Eine Mehrheit (85\%) der heutigen ÄNW-Mitglieder sind auch Grundversorger, von allen im Kantonsgebiet praktizierenden Grundversorger sind dies bereits $43 \%$. ÄNW-Mitglieder sind vorwiegend Fachärzte für Allgemeinmedizin FMH $(46,3 \%)$ und Internisten $(40,8 \%)$, eher männlich $(31,6 \%)$ als weiblich $(22,3 \%)$, zudem jünger (in der Altergruppe unter 50 Jahren 37,4\%) als älter (bei über 59jährigen 21\%) (Abb. 1).

\section{Dimensionen}

Von den 36 geschlossenen Fragen wurden 30 in die Faktoranalyse eingeschlossen. 28 Fragen konnten 4 Dimensionen zugeordnet werden, nicht jedoch die 2 verbleibenden Fragen. Damit ergaben sich folgende Dimensionen (vgl. Tab. 1. für die in den Dimensionen verwendeten Fragen):

- ÄNW und Patientenbetreuung, 7 Fragen, Cronbach $\alpha=0,84$;

- Qualitätssicherung in ÄNW, 7 Fragen, Cronbach $\alpha=0,83$;

- ÄNW und Finanzen, 10 Fragen, Cronbach $\alpha=$ 0,74 ;

- Bereitschaft zur Übernahme von Verantwortung, 4 Fragen, Cronbach $\alpha=0,62$.

Die Auswertung der Antworten pro Dimension zeigt, dass die Fragen aus der Dimension «ÄNW und Patientenbetreuung» am wenigsten positiv beurteilt wurde. Im Durchschnitt erhielten nur 0,87 Fragen von den 7 in dieser Dimension $(12,4 \%$, Tab. 2) eine positive Antwort. Noch am ehesten optimistisch gesehen wird die Frage 


\section{Tabelle 1}

Verteilung der Anteile zustimmender Antworten pro Dimension (100\% = Summe aller positiven Antworten pro Dimension).

Dimension «Patientenbetreuung» (in ÄNW mit und ohne Budgetverantwortung)

\section{Frage Welche Auswirkungen haben ÄNW..}

20

... auf die Qualität der Zusammenarbeit mit den von Ihnen berücksichtigten Konsiliarii/Spitälern/ bei Spezialisten: Zuweisern?

16

19

... auf die Behandlungsqualität von Netzwerkpatienten im Vergleich zu ausserhalb des Modells?

... auf die Auswahl der von Ihnen berücksichtigten Konsiliarii/Spitäler/ bei Spezialisten: auf den Kreis der Zuweiser?

$17 \quad$... auf die Arzt-Patienten-Beziehung?

$5 \quad$...mit Budgetverantwortung auf die Möglichkeit, Ihren Netzwerkpatienten die jeweils wirksamste Behandlung zukommen zu lassen?

18 ... auf Ihre berufliche Autonomie?

29 ... auf die Zeit und den Aufwand für Administration und Controlling?
$\%$ aller positiven Antworten pro Dimension $24,28 \%$

$18,17 \%$

$16,88 \%$

$13,50 \%$

$11,74 \%$

$8,04 \%$

$7,40 \%$

$100 \%$

$\%$ aller positiven Antworten pro Dimension

$20,20 \%$

$17,37 \%$

$16,20 \%$

$15,54 \%$

$15,04 \%$

$7,88 \%$

$7,77 \%$

$100 \%$

$\%$ aller positiven Antworten pro Dimension

$18,21 \%$

$15,06 \%$

$14,99 \%$

$10,35 \%$

$10,10 \%$

$8,21 \%$

$7,48 \%$

$6,75 \%$

$4,68 \%$

$4,16 \%$

$100 \%$

$\%$ aller positiven Antworten pro Dimension

$25,89 \%$

$24,80 \%$

$24,76 \%$

$24,54 \%$

$100 \%$ Abklärungsstrategien systematisch und kritisch zu reflektieren Jeder Arzt ist mitverantwortlich für die Kontrolle der Gesundheitskosten 
nach den «Auswirkungen von ÄNW auf die Qualität der Zusammenarbeit mit den Konsiliarii oder Spitälern» (bzw. bei Spezialisten mit den Zuweisern: Frage 20, die 24\% aller positiven Antworten in dieser Dimension vereinigte). Die Frage nach den Auswirkungen von ÄNW auf die Administration und das Controlling von ÄNWPatienten erhielt am wenigsten positive Stimmen (Frage 29: 7,4\%; Tab. 1). In der Dimension «Qualitätssicherung in ÄNW» wurden im Mittel 2,53 der 7 Fragen positiv beurteilt (36,1\%), am meisten Zustimmung fand die Frage nach der «Bereitschaft, die Praxiskosten innerhalb des ÄNW zu besprechen» (Frage 14: 20,2\%). Eine geringe Akzeptanz zeigte sich für Praxiszertifizierungen (Frage 12), «Qualitätskontrollen im Netzwerk, Fallbesprechungen, QZ usw.» (Frage 31) werden angesichts von nur 7,77\% aller positiven Antworten zur unnötigen Zusatzbelastung. Die finanziellen Aspekte in ÄNW wurden zu 40,2\% positiv beurteilt (4,01 von 10 Fragen). Allerdings fand die Feststellung «Budgetmitverantwortung

\section{Tabelle 2}

Anzahl und Anteil positiv beurteilter Fragen pro Dimension ${ }^{\mathrm{a}}$.

\begin{tabular}{|c|c|c|c|c|}
\hline Dimension & Patientenbetreuung & Qualitätssicherung & Finanzen & Verantwortung \\
\hline Anzahl Fragen ${ }^{\mathrm{b}}$ & 7 & 7 & 10 & 4 \\
\hline \multicolumn{5}{|l|}{ Typ } \\
\hline Alle & $0,87(12,4 \%)$ & $2,53(36,1 \%)$ & $4,02(40,2 \%)$ & $3,23(80,8 \%)$ \\
\hline \multirow[t]{3}{*}{ Netzwerkmitglieder } & 1,4 & 3,2 & 4,9 & 3,4 \\
\hline & $(1,2-1,7)$ & $(2,9-3,5)$ & $(4,6-5,3)$ & $(3,3-3,6)$ \\
\hline & $20,6 \%$ & $45,5 \%$ & $49,3 \%$ & $85,3 \%$ \\
\hline \multirow[t]{3}{*}{ Mitgliedschaft innert 2 Jahren vorgesehen } & 1,1 & 3,1 & $4,2^{*}$ & 3,4 \\
\hline & $(0,9-1,3)$ & $(2,8-3,4)$ & $(3,8-4,5)$ & $(3,3-3,6)$ \\
\hline & $16,3 \%$ & $44,5 \%$ & $41,6 \%$ & $85,1 \%$ \\
\hline \multirow[t]{3}{*}{ Mitgliedschaft nach 2 Jahren vorgesehen } & $0,6^{*}$ & $2,1^{*}$ & $3,8^{*}$ & 3,2 \\
\hline & $(0,4-0,8)$ & $(1,7-2,4)$ & $(3,4-4,1)$ & $(3,0-3,4)$ \\
\hline & $8,5 \%$ & $29,8 \%$ & $37,6 \%$ & $79,8 \%$ \\
\hline \multirow[t]{3}{*}{ Nie Mitgliedschaft vorgesehen } & $0,5^{*}$ & $1,5^{*}$ & $2,7^{*}$ & $2,6^{*}$ \\
\hline & $(0,2-0,7)$ & $(1,1-1,9)$ & $(2,2-3,1)$ & $(2,4-2,8)$ \\
\hline & $6,6 \%$ & $21,4 \%$ & $26,7 \%$ & $65,1 \%$ \\
\hline \multirow[t]{3}{*}{ Hausarzt } & 1 & 2,6 & 4,1 & 3,2 \\
\hline & $(0,8-1,2)$ & $(2,3-2,8)$ & $(3,8-4,4)$ & $(3,1-3,4)$ \\
\hline & $14,5 \%$ & $36,5 \%$ & $41,5 \%$ & $81,0 \%$ \\
\hline \multirow[t]{3}{*}{ Spezialist } & 0,8 & 2,7 & 3,7 & 3,2 \\
\hline & $(0,5-1,2)$ & $(2,3-3,2)$ & $(3,2-4,2)$ & $(2,9-3,4)$ \\
\hline & $12,1 \%$ & $39,0 \%$ & $37,0 \%$ & $79,0 \%$ \\
\hline \multirow[t]{3}{*}{ «andere»c } & 0,9 & 2,7 & 4 & 3,2 \\
\hline & $(0,7-1,2)$ & $(2,3-3,0)$ & $(3,7-4,4)$ & $(3,1-3,4)$ \\
\hline & $13,5 \%$ & $38,2 \%$ & $40,1 \%$ & $80,8 \%$ \\
\hline \multirow[t]{3}{*}{ Ärztinnen } & 1,1 & 2,7 & 3,9 & 3,2 \\
\hline & $(0,9-1,3)$ & $(2,3-3,0)$ & $(3,5-4,4)$ & $(3,0-3,3)$ \\
\hline & $15,7 \%$ & $37,9 \%$ & $39,5 \%$ & $78,9 \%$ \\
\hline \multirow[t]{3}{*}{ Ärzte } & $0,7^{\circ}$ & 2,3 & 3,8 & 3,1 \\
\hline & $(0,6-0,8)$ & $(2,1-2,5)$ & $(3,6-4,0)$ & $(3,1-3,2)$ \\
\hline & $10,3 \%$ & $32,7 \%$ & $38,1 \%$ & $78,7 \%$ \\
\hline \multicolumn{5}{|c|}{$\begin{array}{l}\text { a Anzahl positiv beantworteter Fragen pro Dimension, als Mittelwerte, korrigiert für Alter und Geschlecht. Oben: absolute Werte = least square mean; } \\
\text { unten: Zustimmung zu allen Fragen pro Dimension (in Prozent). }\end{array}$} \\
\hline \multicolumn{5}{|l|}{ b vgl. Tabelle 1 für die einzelnen Fragen. } \\
\hline \multicolumn{5}{|c|}{$\begin{array}{l}\text { c Spezialisten mit mehrheitlich direktem Patientenzugang (Psychiater/ORL/Dermatologen/Augenärzte etc.) - im Gegensatz zu Spezialisten mit mehrheitlich } \\
\text { Konsiliarfunktion. }\end{array}$} \\
\hline \multicolumn{5}{|c|}{${ }^{*}=p<0,05$ im Vergleich zu Netzwerkmitgliedern, ${ }^{\circ}=p<0,05$ im Vergleich zu Ärztinnen. } \\
\hline
\end{tabular}


führt zu einer Abnahme meiner totalen Fallkosten (Summe der direkten Praxiskosten und der veranlassten Kosten)» am wenigsten Zustimmung in dieser Dimension (Frage 7: 4,68\%). Am meisten zugestimmt wurde der Feststellung «ÄNW sollen nur diejenigen Kollegen aufnehmen, die sich vertraglich verpflichten, die ÄNWAnforderungen zu erfüllen» (Frage 22: 18,21\%). Als Dimension erhielt die «Bereitschaft zur Übernahme von Verantwortung» am meisten $\mathrm{Zu}$ stimmung: Im Mittel stimmten 80,8\% zu (3,4 von 4 Fragen).

\section{Zustimmung zu den Dimensionen nach ÄNW-Zugehörigkeit, Funktion und Geschlecht (Tab. 2, Abb. 2)}

ÄNW-Mitglieder weisen für alle untersuchten Dimensionen die höchsten Anteile positiver Antworten pro Dimension auf (\% positiver Antworten pro Dimension, alters- und geschlechtskorrigiert: Patientenbetreuung 20,6\%, Qualitätssicherung 45,5\%, Finanzen 49,3\% und Verantwortung übernehmen 85,3\%), dicht gefolgt von jenen Kolleginnen und Kollegen, die einen Beitritt in den nächsten zwei Jahren planen (Patientenbetreuung 16,3\%, Qualitätssicherung $44,5 \%$, Finanzen $41,6 \%$ und Verantwortung übernehmen 85,1\%). «ÄNW-Gegner» äusserten sich zu allen vier Dimensionen signifikant weniger positiv im Vergleich mit ÄNW-Mitgliedern (mit folgenden Werten: Patientenbetreuung 6,6\%, Qualitätssicherung 21,4\%, Finanzen $26,7 \%$ und Verantwortung übernehmen $65,1 \%)$. Ärzte, die erst nach zwei Jahren einen ÄNW-Beitritt vorsehen, stimmten den Dimensionen Patientenbetreuung mit 8,5\%, Qualitätssicherung mit 29,8\% und Finanzen mit 37,6\% im Vergleich zu ÄNW-Mitgliedern signifikant weniger zu. Dagegen zeigten die Anteile positiver Antworten pro Dimension keine signifikanten Unterschiede nach Funktion (Grundversorger, Konsiliarius, «andere»).

Ärzte schätzten die Dimension «Patientenbetretung im ÄNW» signifikant weniger positiv ein (10,3\% positiver Antworten pro Dimension) als Ärztinnen $(15,7 \%$ positiver Antworten pro Dimension).

\section{Diskussion}

Viele Ärzte - einschliesslich nun auch die FMH, aber ebenso Versicherte, Patienten und Politiker, etwas weniger auch die Versicherer - setzen grosse Hoffnungen in MC und speziell in ÄNW zur Erhaltung einer qualitativ guten und finanzierbaren medizinischen Versorgung. Inwieweit die Berner Ärzteschaft diese Einschätzungen und Hoffnungen teilt, wurde bisher nie systematisch untersucht. Angesichts der negativen Schlagzeilen, die vorwiegend aus MC-Experimenten aus dem angloamerikanischen Raum bis zu uns durchgedrungen sind, muss verständlicherweise mit Bedenken oder sogar Widerstand der hiesigen Ärzte gegenüber MC und ÄNW gerechnet werden [16-18]. Andererseits können angesichts steigender Gesundheitskosten und begrenzter Ressourcen auch von ärztlicher Seite innovative Experimente und Unterstützung erwartet werden. Um diese Entwicklungen einschätzen und allenfalls ÄNW-Gründungen gezielt unterstützen zu können, erteilte die Berner Ärztegesellschaft (zusammen mit dem Berner Trustcenter PonteNova) den Auftrag zu einer Umfrage zu den Meinungen und dem Verhalten der Berner Ärzteschaft gegenüber ÄNW mit und ohne Budgetmitverantwortung.

Die Rücklaufrate dieser Umfrage erreichte knapp 50\% mit 39,9\% auswertbaren Fragebogen. Unter den Teilnehmern sind Grundversorger leicht übervertreten, wahrscheinlich weil sie von ÄNW-Aktivitäten deutlich mehr betroffen sind als «Nichtgrundversorger». Ein Drittel der Teilnehmer ist bereits Mitglied (oder dabei, eine Mitgliedschaft $\mathrm{zu}$ erwerben) sowohl in ÄNW mit wie ohne Budgetmitverantwortung. Da aktuell keine Gesamtdaten bezüglich der Mitgliedschaft der Berner Ärzteschaft in einem ÄNW zur Verfügung stehen, kann nicht überprüft werden, inwiefern unsere Umfrageresultate - im Vergleich zur Gesamtpopulation - einen Bias aufweisen. Da sich die Einschätzungen der in der Umfrage leicht untervertretenen Spezialisten nicht signifikant von jenen der Grundversorger unterscheiden, kann davon ausgegangen werden, dass die Resultate repräsentativ sind. ÄNWMitglieder sind signifikant jünger als Nichtmitglieder, was nicht überrascht, da ältere Kollegen ihre Praxis in naher Zukunft aufgeben werden und daher keinen Handlungsbedarf mehr bezüglich Veränderungen wie einem ÄNW-Beitritt sehen.

Aus der Faktoranalyse der 30 Fragen zur Meinung und Erwartung bezüglich der Auswirkung von ÄNW resultieren vier Dimensionen, was die ursprünglichen Intentionen der Fragebogenentwicklung zumindest teilweise widerspiegelt. Die Berner Ärzteschaft befürchtet am meisten, dass sich ÄNW negativ auf die Qualität der Patientenbetreuung auswirken werden. Bell et al. [19] haben bereits vor Jahren unter amerikanischen Ärzten eine vergleichbare Einschätzung gefunden. Es bestehen somit auch bei uns grosse Zweifel, inwiefern ÄNW tatsächlich die postulierten Vorteile bringen, wie etwa «auf die Bedürfnisse der Patientinnen und Patienten ausgerichtete 
Abbildung 2

Box-Plot mit der mittleren Anzahl positiv beurteilter Fragen pro Dimension.
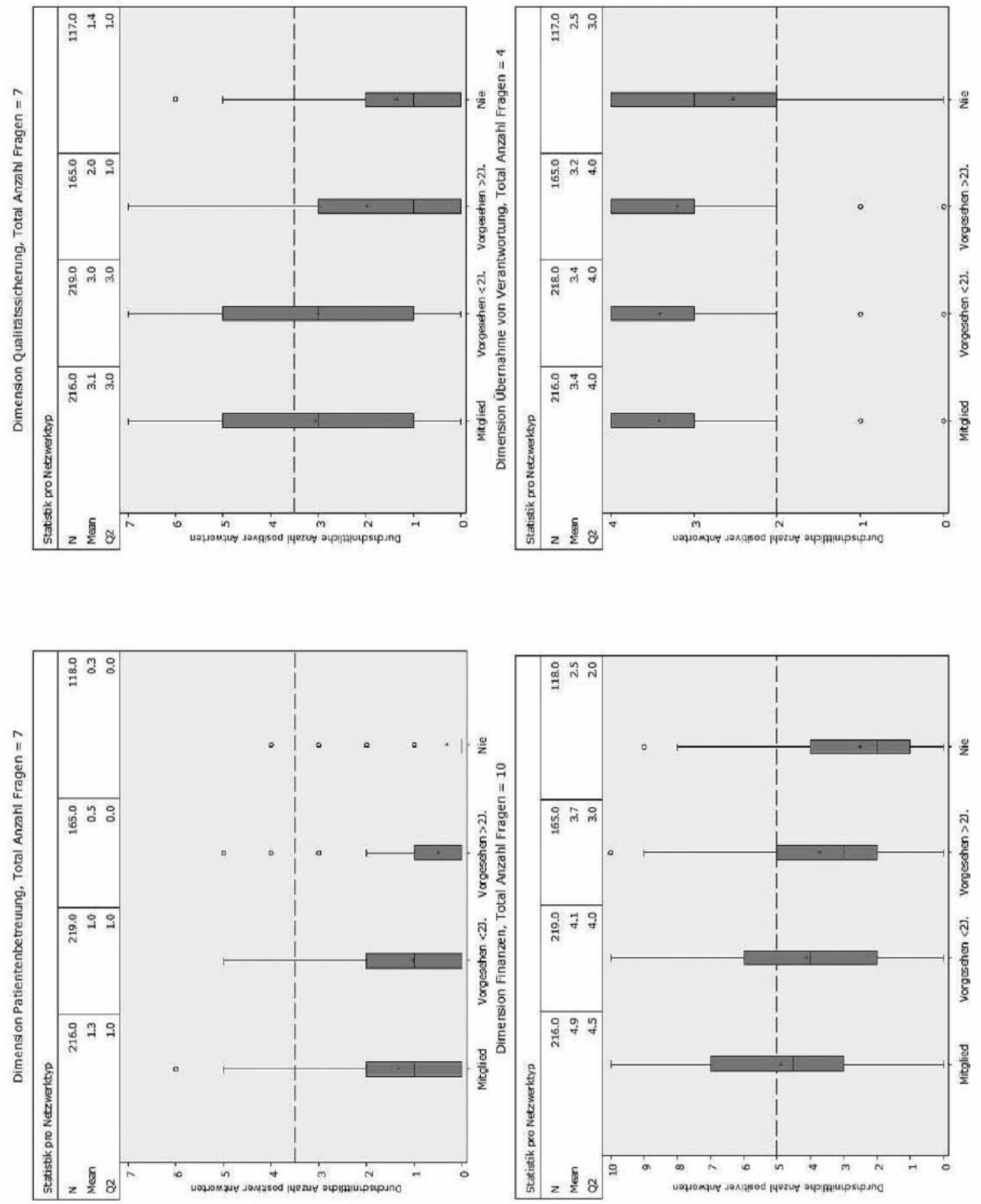

Q2 / * =Median; - = Mean; oberes Ende des Kastens = höchste Quartile; unteres Ende des Kastens = tiefste Quartile; $T$ = oberer Querstrich dritte Quartile plus 1,5 $\times$ den Interquartilbereich $(\mathrm{IQR}) ; \perp=$ unterer Querstrich: erste Quartile 1,5 $\times$ den Interquartilbereich $(\mathrm{IQR})$; $\mathrm{o}=$ Extremwerte. 
Gesundheitsleistungen», «vereinbarte Behandlungsprozesse» oder «eine gemeinsame Betreuungskultur» [21].

Der vermutete Verlust der beruflichen Autonomie in der Patientenbetreuung (Frage 18) und die Angst vor (noch mehr) Zeitverschwendung für Administration und Controlling (Frage 29) scheinen dabei eine Hauptrolle zu spielen. Obwohl die «Behandlungsqualität im ÄNW» am zweitmeisten positive Antworten auf sich summiert (Frage 16: 18,17\% der positiven Antworten in dieser Dimension), steht diese geringe $\mathrm{Zu}$ stimmung in deutlichem Widerspruch zur vorhandenen Evidenz, die deutlich bessere Patientenresultate $[7,13,14]$ und höhere Qualitäten in MC-Modellen [15] im Vergleich zu Systemen mit Einzelleistungsabgeltung (fee-for-service) zeigt.

Auffallend ist dabei, dass auch ÄNW-Mitglieder diese Dimension kritisch beurteilen. Dabei bleibt offen, ob ihr Urteil wegen fehlender oder erst weniger Erfahrungen eher eine Erwartungshaltung wiedergibt oder sich bereits auf eigene Erfahrungen im ÄNW abstützt. Die Perspektive der Budgetmitverantwortung mag dabei eine Rolle spielen, obwohl die Mehrzahl der ÄNW im Kanton Bern heute (noch) ohne laufen. Dass Ärztinnen die Patientenbetreuung in ÄNW optimistischer als ihre männlichen Kollegen beurteilen, mag vielleicht eine feminisierte Sicht gemeinsamer Betreuung, möglicherweise auch eine geringere Angst vor einem Autonomieverlust widerspiegeln. Die beiden Dimensionen «Qualitätssicherung» und «Finanzen» in ÄNW erfuhren mehr oder weniger Zustimmung, grundsätzlich wurden diese Aspekte neutral beurteilt. Gründe dafür können sein, dass beide Themen im Zentrum der (politischen) Diskussionen stehen, und dies unabhängig von der Bildung von ÄNW. Die diesbezüglichen Auswirkungen von ÄNW werden von der Ärzteschaft weder als umwerfend positiv noch als sehr negativ beurteilt. Auch hier zeigt sich unter Mitgliedern der höchste und unter «ÄNW-Gegnern» der niedrigste Grad an Zustimmung. Überwältigende Zustimmung fand einzig die Dimension «Bereitschaft zur Übernahme von Verantwortung», beispielsweise zu einem «sorgfältigeren Umgang mit den finanziellen Ressourcen im Gesundheitswesen», «zur Vermeidung von unnötigen Abklärungen» oder «gemeinsam mit Kollegen im ÄNW seine Behandlungs- und Abklärungsstrategien systematisch und kritisch zu reflektieren».
Dass dies nicht nur unverbindliche Lippenbekenntnisse sind, sondern im ÄNW mit einem Tatbeweis einhergehen (werden), zeigt sich an der doch überraschenden Tatsache, dass unter allen Aspekten zur Qualitätssicherung «die Bereitschaft zur Diskussion der Praxiskosten im ÄNW» zuoberst in der Gunst der Ärzteschaft - genauer der Mehrheit, bestehend aus aktuellen und zukünftigen ÄNW-Mitgliedern - steht, noch vor der «Bereitschaft, dem ÄNW medizinische Daten zur Verfügung zu stellen» oder «an einer minimalen Zahl an obligatorischen Qualitätszirkeln teilzunehmen». Damit zeigt die Berner Ärzteschaft, dass sie - trotz der Zurückhaltung gegenüber ÄNW - einige Grundbedingungen von MC in ihrem Alltag bereits akzeptiert (hat), wie beispielsweise Qualitätszirkel oder die Notwendigkeit der Transparenz. Zum Prüfstein von ÄNW wird die Übernahme von Budgetmitverantwortung werden, da diese kaum erkennbare Vorteile bringt und selbst von einer Mehrheit von Netzwerkbefürwortern abgelehnt wird.

\section{Schlussfolgerungen}

Ende 2006 war ein Drittel der Berner Ärzteschaft mit Praxisbewilligung Mitglied in einem strukturierten ÄNW, und mindestens weitere 50\% gedenken in den nächsten Jahren einem ÄNW beizutreten. Zusammenfassend zeigt die Umfrage, dass Mitglieder von ÄNW die positivsten Einstellungen, Erfahrungen/Meinungen und Erwartungen bezüglich ÄNW haben und dass diese abnehmen, je weiter ein Beitritt in die Ferne rückt. Kolleginnen und Kollegen, die nie einem ÄNW beizutreten gedenken, haben folgerichtig die diesbezüglich negativste Einschätzung. Funktion und Facharzttitel haben auf diese Urteile keinen, das Geschlecht nur einen partiellen Einfluss. Die grössten Zweifel bezüglich negativer Auswirkungen von ÄNW betreffen die Qualität der Patientenbetreuung, während Massnahmen zur Qualitätssicherung und finanzielle Aspekte tendenziell neutral beurteilt werden. ÄNW und Promotoren von ÄNW bleibt damit die Hauptaufgabe, die möglichen negativen Auswirkungen von ÄNW und Budgetmitverantwortung speziell auf die Patientenbetreuung - sachlich zu diskutieren und durch Fakten zu widerlegen. Die von uns gefundenen Resultate sollten durch eine gleiche Studie - in einem andern Kanton mit weiter fortgeschrittenen ÄNW- oder MC-Aktivitäten - verifiziert oder falsifiziert werden. 


\section{Literatur}

1 Huerta TR, Casebeer A, Vanderplaat M. Using networks to enhance health services delivery: perspectives, paradoxes and propositions. Healthc Pap. 2006;7(2):10-26.

2 Morrison EW. Newcomers relationships: the role of social network ties during socialization. Acad Management J. 2002;45:1149-60.

3 Bindman AB, Weiner JP, Majeed A. Primary care groups in the United Kingdom: quality and accountability. Health Aff (Millwood). 2001; 20(3):132-45.

4 Endslay S, Kirkegaar M, Linares A. Working together: communities of practice in family medicine. Fam Pract Manag. 2005;12(1):28-32.

5 Hayward S. Networks: better language required. Healthc Pap. 2006;7(2):62-6; discussion 8-75.

6 Smith AL, Epstein AL. Improving performance in a contracted physician network. Physician Exec. 1999;25(6):60-3.

7 Bodenheimer T, Wagner EH, Grumbach K. Improving primary care for patients with chronic illness: the chronic care model, Part 2. JAMA. 2002; 288(15):1909-14.

8 Wagner EH, Austin BT, Von Korff M. Organizing care for patients with chronic illness. Milbank Q. 1996;74(4):511-44.

9 The European definition of general practice. www.woncaeurope.org. 2002.

10 McGuire T. Handbook of Health Economics. Physician agency; 2000.

11 Forrest CB. Primary care in the United States: primary care gatekeeping and referrals: effective filter or failed experiment? BMJ. 2003;326(7391):692-5.

12 Redfern J, Bowling A. Efficiency of care at the primary-secondary interface: variations with GP fund holding. Health Place. 2000;6(1):15-23.

13 Grimshaw JM, Shirran L, Thomas R, et al. Changing provider behavior: an overview of systematic reviews of interventions. Med Care. 2001;39(8 Suppl 2):II2-45.
14 Weingarten SR, Henning JM, Badamgarav E, et al. Interventions used in disease management programmes for patients with chronic illness - which ones work? Meta-analysis of published reports. BMJ. 2002;325(7370):925.

15 Roohan PJ, Butch JM, Anarella JP, Gesten F, Shure K. Quality measurement in medicaid managed care and fee-for-service: the New York State experience. Am J Med Qual. 2006;21(3):185-91.

16 Huby G, Gerry M, McKinstry B, Porter M, Shaw J, Wrate R. Morale among general practitioners: qualitative study exploring relations between partnership arrangements, personal style, and workload. BMJ. 2002;325(7356):140.

17 Behrens J. [Physician's anxiety and physician's elegance. Problems in dealing with cost reduction, education of general practitioners and optimal size of practice networks in a cross-national comparison]. Gesundheitswesen. 2000;62(3):130-7.

18 Meyer PC, Denz MD. [Social change in the physician's role and medical practice caused by managed care in Switzerland]. Gesundheitswesen. 2000;62(3):138-42.

19 Bell RA, Kravitz RL, Siefkin AD, Foulke GE. Physicians' attitudes toward managed care: assessment and potential effects on practice behaviors. Am J Manag Care. 1997;3(9):1297-304.

20 Raetzo MA. [Continuing education, motivation for cost control, a Swiss experiment]. Gesundheitswesen. 2000;62(3):143-7.

21 Stricker B. Ärztenetzwerke - Klärung eines Begriffs. Schweiz Ärztezeitung. 2005;86(15):883-7.

22 Thesenpapier FMH. Managed Care - Kontrahierungszwang. Schweiz Ärztezeitung. 2006;87(36): 1537-40.

23 Vorstand der Ärztegesellschaft des Kantons Bern. Offener Brief an den VSAO: Wir unterstützen Euch! DOCBE. 2006;6:7.

24 Custer R, Scarcella, JA, Stewart, BR. The modified Delphi technique - a rotational modification. J Vocational Tech Educ. 1999;15(2). 